\title{
SOME BESSEL FUNCTION EXPANSIONS
}

BY W. H. WISE

This is a formal derivation of some asymptotic Bessel function expansions. An interesting feature of the derivation is that it makes use of Heaviside's generalized exponential series. ${ }^{*}$ The chief results arrived at, the Hankel function expansions (4) and (6), are generalizations of formulas (5) and (8) on page 141 in Watson's Theory of Bessel Functions in the same sense that Heaviside's generalized exponential series is a generalization of the ordinary exponential series.

In the course of some work on dipole radiation the author stumbled onto a particular case of the following relation

$$
H_{0}^{(1)}\left(z(1-a)^{1 / 2}\right)=\sum_{n=-\infty}^{n=\infty} \frac{(a z / 2)^{n-\nu}}{\Pi(n-\nu)} H_{n-\nu}^{(1)}(z) .
$$

In the particular case where $\nu$ is $1 / 2, a$ is in the fourth quadrant and $z$ is in the first quadrant and has a large real part. Except for a missing common factor, the left-hand side in the particular case is half the Zenneck wave component of the wave function (as measured at the surface of the earth) for a vertical dipole centered on the interface between air and earth, the descending half of the series is the negative of the sky wave component of the wave function and the ascending part of the series is the convergent expansion for half the Zenneck wave component plus the sky wave component.

The suggestion immediately offered itself that (1) should hold for all values of $\nu$, not just for $\nu=1 / 2$.

If we assume for the moment that (1) holds for all $\nu$, and differentiate with respect to $z$, we get

$$
\begin{aligned}
& H_{1}^{(1)}\left(z(1-a)^{1 / 2}\right)=\frac{-1}{(1-a)^{1 / 2}} \sum_{n=-\infty}^{n=\infty} \frac{(a z / 2)^{n-\nu}}{\Pi(n-\nu)} H_{n-\nu-1}^{(1)}(z) \\
& =\frac{-1}{(1-a)^{1 / 2}} \sum_{n=-\infty}^{n=\infty} \frac{(a z / 2)^{n-\nu}}{\Pi(n-\nu)} \cdot\left[\frac{2(n-\nu)}{z} H_{n-\nu}^{(1)}(z)-H_{n-\nu+1}^{(1)}(z)\right]
\end{aligned}
$$
792.

* See John R. Carson, Transactions of this Society, vol. 31 (1929), pp. 782- 
or

(2) $H_{1}^{(1)}\left(z(1-a)^{1 / 2}\right)=(1-a)^{1 / 2} \sum_{n=-\infty}^{n=\infty} \frac{(a z / 2)^{n-\nu}}{\Pi(n-\nu)} H_{n-\nu+1}^{(1)}(z)$.

By use of the recurrence relation for Bessel functions,

$$
Z_{p+1}(z)=\frac{2 p}{z} Z_{p}(z)-Z_{p-1}(z)
$$

we get from (1) and (2)

$$
\begin{aligned}
H_{2}^{(1)}\left(z(1-a)^{1 / 2}\right) & =\sum_{n=-\infty}^{n=\infty} \frac{(a z / 2)^{n-\nu}}{\Pi(n-\nu)}\left[\frac{2}{z} H_{n-\nu+1}^{(1)}(z)-H_{n-\nu}^{(1)}(z)\right] \\
= & \sum_{n=-\infty}^{n=\infty} \frac{(a z / 2)^{n-\nu}}{\Pi(n-\nu)}\left[H_{n-\nu+2}^{(1)}(z)-(n-\nu) \frac{2}{z} H_{n-\nu+1}^{(1)}(z)\right] \\
= & \sum_{n=-\infty}^{n=\infty} \frac{(a z / 2)^{n-\nu}}{\Pi(n-\nu)} H_{n-\nu+2}^{(1)}(z) \\
& \quad-a \sum_{n=-\infty}^{n=\infty} \frac{(a z / 2)^{n-\nu-1}}{\Pi(n-\nu-1)} H_{n-\nu+1}^{(1)}(z) \\
= & (1-a) \sum_{n=-\infty}^{n=\infty} \frac{(a z / 2)^{n-\nu}}{\Pi(n-\nu)} H_{n-\nu+2}^{(1)}(z),
\end{aligned}
$$

and it is now more or less evident that in general

$$
\text { (4) } \quad H_{m}^{(1)}\left(z(1-a)^{1 / 2}\right)=(1-a)^{m / 2} \sum_{n=-\infty}^{n=\infty} \frac{(a z / 2)^{n-\nu}}{\Pi(n-\nu)} H_{n-\nu+m}^{(1)}(z) \text {. }
$$

Equation (4) is one of a pair of similar formulas. If, in place of (1), we start out with the idea that

$$
H_{0}^{(1)}\left(z(1+a)^{1 / 2}\right)=\sum_{n=-\infty}^{n=\infty} \frac{(a z / 2)^{n-\nu}}{\Pi(n-\nu)} H_{-(n-\nu)}^{(1)}(z),
$$

then steps identical with those taken in going from (1) to (4) lead us to

(6) $\quad H_{-m}^{(1)}\left(z(1+a)^{1 / 2}\right)=(1+a)^{m / 2} \sum_{n=-\infty}^{n=\infty} \frac{(a z / 2)^{n-\nu}}{\Pi(n-\nu)} H_{-(n-\nu+m)}^{(1)}(z)$,

for integral values of $m$. 
On page 180 in Watson's Theory of Bessel Functions we find the relation

$$
H_{p}^{(1)}(z)=\frac{e^{-i(\pi / 2) p}}{i \pi} \int_{-\infty-i \pi / 2}^{\infty+i \pi / 2} e^{i z \cosh w-p w} d w
$$

good for all values of $p$ and for $|\arg z|<\pi / 2$.

By setting ( 7$)$ in the right-hand side of (6), we get

(a) $(1+a)^{m / 2} \sum_{n=-\infty}^{n=\infty} \frac{(a z / 2)^{n-\nu}}{\Pi(n-\nu)} \frac{1}{i \pi} \int e^{i z \cosh w-(w+i \pi / 2)(-n+\nu-m)} d w$,

(b) $=(1+a)^{m / 2} \frac{1}{i \pi} \int e^{i z \cosh w+m(w+i \pi / 2)} \sum_{n=-\infty}^{n=\infty} \frac{\left(\frac{a z}{2} e^{w+i \pi / 2}\right)^{n-\nu}}{\Pi(n-\nu)} d w$.

Now it is known that

$$
\sum_{n=-\infty}^{n=\infty} \frac{z^{n-\nu}}{\Pi(n-\nu)}
$$

is a formal series representation for $e^{z}$; it is Heaviside's generalized exponential series. So the series in the integrand can be summed. The integral now is

$$
\begin{aligned}
(1+a)^{m / 2} \frac{1}{i \pi} \int e^{i z \cosh w+m(w+i \pi / 2)} \exp \left(\frac{a z}{2} e^{w+i \pi / 2}\right) d w \\
(\mathrm{c}) \quad=(1+a)^{m / 2} \frac{1}{i \pi} \int e^{i z \cosh w} \sum_{n=0}^{\infty} \frac{1}{n !}\left(\frac{a z}{2}\right)^{n} e^{(n+m)(w+i \pi / 2)} d w \\
=(1+a)^{m / 2} \sum_{n=0}^{\infty} \frac{(a z / 2)^{n}}{n !} H_{-m-n}^{(1)}(z)=H_{-m}^{(1)}\left(z(1+a)^{1 / 2}\right) .
\end{aligned}
$$

The right-hand side of (4) may be summed in the same way. The series in (b) is a good representation for $\exp \left((a z / 2) e^{w+i \pi / 2}\right)$ over only a part of the integration range, but, since the series can be recognized where it is valid, its sum may be used where the series is not valid. Because the series in (b) is a good representation for $\exp \left((a z / 2) e^{w+i \pi / 2}\right)$ over only a part of the integration range the integral (b) is meaningless except as it is interpreted as (a) or (c). This formal summation of the righthand sides of (4) and (6) is therefore not entirely satisfactory. The following argument will be more convincing and inciden- 
tally indicates that by the application of sufficient ingenuity the steps from (a) to (b) and (b) to (c) could be justified.

Since the right-hand side of (8) is a solution of Bessel's equation it must be possible to determine constants $A$ and $B$ such that

$$
\begin{aligned}
& A H_{m}^{(1)}\left(z(1-a)^{1 / 2}\right)+B J_{m}\left(z(1-a)^{1 / 2}\right) \\
& \quad=(1-a)^{m / 2} \sum_{n=-\infty}^{n=\infty} \frac{(a z / 2)^{n-\nu}}{\Pi(n-\nu)} H_{n-\nu+m}^{(1)}(z) .
\end{aligned}
$$

The right-hand side of (8) and the Bessel functions on the left satisfy the partial differential equation

$$
2(1-a) \frac{\partial u}{\partial a}+z \frac{\partial u}{\partial z}=0 .
$$

It follows that $A$ and $B$ are independent of $a$.

To see that $A=1$ and $B=0$, let $z \rightarrow \infty$ and $a \rightarrow 0$ while $-a z i / 2$ keeps some large constant value such that Heaviside's generalized exponential series is valid. Then each $H_{p}^{(1)}(z)$ may be replaced by the first term of the expansion

$$
\begin{aligned}
& H_{p}^{(1)}(z) \sim\left(\frac{2}{\pi z}\right)^{1 / 2} e^{i(z-(2 p+1) \pi / 4)}\left(1+i \frac{4 p^{2}-1}{1 ! 8 z}\right. \\
&\left.-\frac{\left(4 p^{2}-1\right)\left(4 p^{2}-3^{2}\right)}{2 ! 8^{2} z^{2}}+\cdots\right),
\end{aligned}
$$

and the principal terms in (8) are, after canceling the common factor $(2 /(\pi z))^{1 / 2}$,

$$
\begin{aligned}
A e^{i[z(1-a / 2)-(2 m+1) \pi / 4]}+ & B \cos \left[z\left(1-\frac{a}{2}\right)-\frac{2 m+1}{4} \pi\right] \\
& =\sum_{n=-\infty}^{n=\infty} \frac{(-a z i / 2)^{n-\nu}}{\Pi(n-\nu)} e^{i(z-(2 m+1) \pi / 4)} \\
& =e^{i[z(1-a / 2)-(2 m+1) \pi / 4]} .
\end{aligned}
$$

It follows that $A=1$ and $B=0$.

If the $H_{n-\nu+m}^{(1)}(z)$ in (8) are replaced by $J_{n-\nu+m}(z)$, then the right-hand side of (9) is replaced by 


$$
\begin{gathered}
\sum_{n=-\infty}^{n=\infty} \frac{(a z / 2)^{n-\nu}}{\Pi(n-\nu)} \cos \left[z-\frac{2(n-\nu+m)+1}{4} \pi\right] \\
=\sum_{n=-\infty}^{n=\infty}\left\{\frac{(-a z i / 2)^{n-\nu}}{\Pi(n-\nu)} \cdot \frac{e^{i(z-(2 m+1) \pi / 4)}}{2}\right. \\
\left.\quad+\frac{(a z i / 2)^{n-\nu}}{\Pi(n-\nu)} \cdot \frac{e^{-i(z-(2 m+1) \pi / 4)}}{2}\right\} \\
=\frac{1}{2} e^{i[z(1-a / 2)-(2 m+1) \pi / 4]}+\frac{1}{2} e^{-i[z(1-a / 2)-(2 m+1) \pi / 4]} \\
=\cos \left[z\left(1-\frac{a}{2}\right)-\frac{2 m+1}{4} \pi\right]
\end{gathered}
$$

and we must conclude that $A=0$ and $B=1$.

The differential equation satisfied by

$$
\begin{aligned}
y & =H_{m}^{(1)}\left(z(1-a)^{1 / 2}\right)-(1-a)^{m / 2} \sum_{n=-\infty}^{n=-N-1} \frac{(a z / 2)^{n-\nu}}{\Pi(n-\nu)} H_{n-\nu+m}^{(1)}(z) \\
& =(1-a)^{m / 2} \sum_{n=-N}^{n=\infty} \frac{(a z / 2)^{n-\nu}}{\Pi(n-\nu)} H_{n-\nu+m}^{(1)}(z)
\end{aligned}
$$

is

$$
\begin{aligned}
y^{\prime \prime}+\frac{y^{\prime}}{z} & +\left(1-a-\frac{m^{2}}{z^{2}}\right) y \\
& =a(1-a)^{m / 2} \frac{(a z / 2)^{-N-\nu-1}}{\Pi(-N-1-\nu)} H_{-N-1-\nu+m}^{(1)}(z) .
\end{aligned}
$$

This differential equation has two particular integrals, $\sum_{-N}^{+\infty}$ and $-\sum_{-\infty}^{-N-1}$, and they differ by the complementary function $H_{m}^{(1)}\left(z(1-a)^{1 / 2}\right)$. The divergent particular integral and the complementary function are inextricably tangled up in the convergent particular integral.

If we choose a sector of the complex plane where $H_{p}^{(1)}(z)$ vanishes as $z$ goes to infinity we can see by inspection of the differential equation that as $z$ goes to infinity the particular integral may behave in either one of two different ways. It may approach zero as does the right-hand side of the differential equation. If it does not approach zero as does the right-hand 
side, it must be approaching a Bessel function in such a way that it does not completely satisfy Bessel's equation, but does so with a small and rapidly vanishing residue, the right-hand side of (10). The divergent particular integral must be the one that vanishes like the right-hand side. The convergent particular integral must be the one that eventually behaves like a Bessel function.

If $H^{(1)}$ is replaced by $J$ the reasoning must be altered to specify that the imaginary part of $z$ remains finite while the real part of $z$ goes to infinity.

Formulas (4) and (6) are generalizations of formulas (5) and (8) on page 141 in Watson. The formulas in Watson are obtained by making $\nu$ zero, or any integer.

It should be noted that in verifying (4) and (6) it was not necessary to require that $m$ be an integer. However, the restrictions on Watson's $\nu$ must apply to $m$ also.

If in (4) we put $m=-1 / 2$ and replace $H^{(1)}$ by $J$, we get, since $\cos x=(\pi x / 2)^{1 / 2} J_{-1 / 2}(x)$,

$$
\left(\frac{2}{\pi z}\right)^{1 / 2} \cos \left(z(1-a)^{1 / 2}\right)=\sum_{n=-\infty}^{n=\infty} \frac{(a z / 2)^{n-\nu}}{\Pi(n-\nu)} J_{n-\nu-1 / 2}(z) .
$$

If in (6) we put $m=-1 / 2$ and replace $H^{(1)}$ by $J$, we get, since $\sin x=(\pi x / 2)^{1 / 2} J_{1 / 2}(x)$,

$$
\left(\frac{2}{\pi z}\right)^{1 / 2} \sin \left(z(1+a)^{1 / 2}\right)=\sum_{n=-\infty}^{n=\infty}-\frac{(a z / 2)^{n-\nu}}{\Pi(n-\nu)} J_{-(n-\nu-1 / 2)}(z) .
$$

If we put $a z / 2=t$, then (11) and (12) are

$$
\begin{aligned}
& \left(\frac{2}{\pi z}\right)^{1 / 2} \cos \left(z^{2}-2 z t\right)^{1 / 2}=\sum_{n=-\infty}^{n=\infty} \frac{t^{n-\nu}}{\Pi(n-\nu)} J_{n-\nu-1 / 2}(z) \\
& \left(\frac{2}{\pi z}\right)^{1 / 2} \sin \left(z^{2}+2 z t\right)^{1 / 2}=\sum_{n=-\infty}^{n=\infty} \frac{t^{n-\nu}}{\Pi(n-\nu)} J_{-(n-\nu-1 / 2)}(z) .
\end{aligned}
$$

Equations (13) and (14) are generalizations of the Glaisher formulas (3) and (4) on page 140 in Watson. The Glaisher formulas are obtained by making $\nu$ zero, or any integer.

If in (11) we put $\nu=1 / 2$, we get

$$
\left(\frac{2}{\pi z}\right)^{1 / 2} \cos \left(z(1-a)^{1 / 2}\right)=\sum_{n=-\infty}^{n=\infty} \frac{(a z / 2)^{n-1 / 2}}{\Pi(n-1 / 2)} J_{n-1}(z)
$$


or

$$
\begin{gathered}
a^{1 / 2} \cos \left(z(1-a)^{1 / 2}\right)=a z J_{0}(z)+\left(\frac{a^{2} z^{2}}{1 \cdot 3}-1\right) J_{1}(z) \\
+\left(\frac{a^{3} z^{3}}{1 \cdot 3 \cdot 5}-\frac{1}{a z}\right) J_{2}(z)+\left(\frac{a^{4} z^{4}}{1 \cdot 3 \cdot 5 \cdot 7}-\frac{1 \cdot 3}{a^{2} z^{2}}\right) J_{3}(z) \\
+\left(\frac{a^{5} z^{5}}{1 \cdot 3 \cdot 5 \cdot 7 \cdot 9}-\frac{1 \cdot 3 \cdot 5}{a^{3} z^{3}}\right) J_{4}(z)+\cdots
\end{gathered}
$$

It will be observed that the left-hand side of (16) is an even function of $z$ while the right-hand side is an odd function of $(z)$. This merely indicates that $|\arg z|<\pi / 2$ and is not surprising.

As a matter of curiosity (16) was computed with $z=10$ and $a=1$. The ascending part of the series, from $J_{0}$ to $J_{15}$ inclusive, is 1.0668. The descending part of the series, from $-J_{1}$ on, is

$$
\begin{array}{llllll}
-0.0435 & -0.0255 & -0.0018 & +0.0033 & +0.0025 & +0.0001 \\
-0.0023 & -0.0043 & -0.0059 & -0.0072 & -0.0081 & -0.0087
\end{array}
$$

and so on. The sum of the first seven terms is -0.0671 , which is a better check than one would expect from these figures The decision as to where to terminate the asymptotic series is complicated by the two changes in sign.

If in (12) we put $\nu=1 / 2$, we get

$$
\left(\frac{2}{\pi z}\right)^{1 / 2} \sin \left(z(1+a)^{1 / 2}\right)=\sum_{n=-\infty}^{n=\infty} \frac{(a z / 2)^{n-1 / 2}}{\Pi(n-1 / 2)} J_{-n+1}(z)
$$

or

$$
\begin{gathered}
a^{1 / 2} \sin \left(z(1+a)^{1 / 2}\right)=a z J_{0}(z)-\left(\frac{a^{2} z^{2}}{1 \cdot 3}-1\right) J_{1}(z) \\
+\left(\frac{a^{3} z^{3}}{1 \cdot 3 \cdot 5}-\frac{1}{a z}\right) J_{2}(z)-\left(\frac{a^{4} z^{4}}{1 \cdot 3 \cdot 5 \cdot 7}-\frac{1 \cdot 3}{a^{2} z^{2}}\right) J_{3}(z)+\cdots
\end{gathered}
$$

With $z=10$ and $a=1$, and so using the terms computed for (16), we find that the left-hand side of (18) is 1.0000 , the ascending part of the series is 0.9786 , the asymptotic part is 0.0230 , and the right side is 1.0016 .

Since both sides of (18) are odd functions of $z$, this formula is only restricted to large absolute values of $z$.

Bell Telephone Laboratories 